\title{
Elevated enolase and caveolin-1 in the heart of rats following dexamethasone-induced toxicity
}

\author{
QUAN-YONG HUANG ${ }^{1}$, XUE-FANG LI $^{2}$ and SHUI-PING LIU ${ }^{3}$ \\ ${ }^{1}$ Department of Pathology, School of Basic Medical Sciences, Dali University, Dali, Yunnan 671000; \\ ${ }^{2}$ School of Basic Medical Sciences, Dali University, Dali, Yunnan 671000; ${ }^{3}$ Department of Forensic Pathology, \\ Zhongshan School of Medicine, Sun Yat-Sen University, Guangzhou, Guangdong 510080, P.R. China
}

Received December 8, 2011; Accepted February 28, 2012

DOI: $10.3892 / \mathrm{mmr} .2012 .817$

\begin{abstract}
Dexamethasone (DEX)-induced heart damage is associated with enzyme and protein alterations. The purpose of this study was to investigate DEX-induced alterations in cardiac enolase and caveolin-1 (cav-1) following DEX administration. Male Wistar rats were randomly divided into two groups: a control and a DEX. The DEX group intraperitoneally received $\mathrm{DEX}$ at the single dose of $10 \mathrm{mg} / \mathrm{kg}$ for 7 consecutive days, and the control was given the same amount of saline via the same route. On day 8 , the rats were anesthetized, and a thoracotomy was performed in all animals. Immunohistochemical analysis was performed to evaluate protein expression of enolase and cav-1. Sections were analyzed by digital image analysis. Our results demonstrated that cardiac protein expression of enolase and cav-1 was altered following DEX-induced toxicity in the rat. The expression of enolase and cav-1 was significantly increased after DEX treatment, supported by integrated optical density compared with the control $(\mathrm{P}<0.05)$. In conclusion, following DEX-induced toxicity, protein expression of enolase and cav-1 was significantly elevated. The current findings indicate that such alterations would be reflected in abnormal cardiac function, and the proteins identified in this study may be useful in revealing the mechanisms underlying DEX-induced toxicity and also in providing various clues for further research.
\end{abstract}

\section{Introduction}

Corticosteroids are hormonal substances widely used in human and veterinary medicine for their anti-inflammatory properties. Among the numerous existing artificial corticosteroids, dexamethasone (DEX) remains the most commonly used, mainly through esterified forms such as acetate or

Correspondence to: Dr Quan-Yong Huang, Department of Pathology, School of Basic Medical Sciences, Dali University, Dali, Yunnan 671000, P.R. China

E-mail: hqy0726@126.com

Key words: enolase, caveolin-1, dexamethasone-induced heart damage phosphate (1). DEX is a potent, synthetic member of the glucocorticoid class of steroid drugs with pleiotropic effects on multiple signaling pathways, and has been widely used in numerous disorders during the last 50 years (2). Recent studies have demonstrated that DEX is routinely prescribed to reduce cerebral oedema and is used increasingly as an anti-emetic (3). It has been reported that nausea, fatigue, and pain scores were all reduced using DEX treatment (4), and a single administration of a DEX implant significantly reduced inflammation in an animal model (5). DEX pretreatment arterializes venous endothelial cells by inducing MAP kinase phosphatase-1 and may protect the veins from inflammation (6).

However, it has also been reported that a number of side effects are attributable to DEX (7). DEX has been shown to cause hyperglycaemia (3), and it has been suggested that it increases the risk of infection in multiple myeloma patients (8). Moreover, intratympanic DEX injection may be a simple and effective treatment for patients with sudden sensorineural hearing loss (9). Yet, recent studies have demonstrated that DEX led to growth retardation, increased mortality and severe kidney damage (10). In addition, DEX treatment leads to a decreased number of sialic acids on the surfaces of human macrophages promoting recognition and uptake of apoptotic cells (11).

It has been shown that DEX-induced toxicity is involved in changes in tropoelastin (12), $\mathrm{Ca}^{2+}$ and $\mathrm{Na}^{+}$(13). However, little is known concerning the changes in enolase and caveolin-1 (cav-1) expression in the heart following DEX-induced toxicity.

The present study utilized immunohistochemistry to evaluate the expression of enolase and cav-1 in the heart, and to determine whether protein expression is altered following DEX-induced toxicity.

\section{Materials and methods}

Sixteen healthy adult male Wistar rats, weighing 210-250 g, were used in this study. All animals were kept in individual stainless steel cages under standardized conditions (constant temperature and humidity, 12-h light-dark cycle). Rats were fed with a standard pellet chow and water throughout the experimental period. All procedures described in this study were approved by the Ethics Committee of Sun Yat-Sen University. 
Animals and study design. Animals were divided randomly into 2 groups $(n=8)$. The experimental group received intraperitoneal injections of $10 \mathrm{mg} / \mathrm{kg}$ DEX (dexamethasone phosphate dissolved in $0.9 \%$ saline) for 7 consecutive days. The control group was injected with $0.9 \%$ saline in equal volumes as the experimental group (14). One day after the last injection, animals were sacrificed by decapitation under ether anesthesia. A thoracotomy was subsequently performed, and the heart was harvested, fixed in phosphatebuffered $10 \%$ formalin, embedded in paraffin wax, and sectioned $(4 \mu \mathrm{m})$ for light microscopy and immunohistochemical examination.

Histopathological examination. Heart specimens from each group were examined histopathologically. After the heart tissues were fixed in $10 \%$ formalin solution for $48 \mathrm{~h}$ and embedded in parafin wax, they were sectioned $(4 \mu \mathrm{m})$, and then stained with hematoxylin and eosin. The slides were coded and semi-quantitative analysis of the sections was performed without knowledge of the treatment protocol. Pathological changes were evaluated in the tissues as previously described $(15,16)$.

Tissue sections and immunohistochemical staining. All rat hearts were immersed in $4 \%$ formaldehyde buffered with phosphate-buffered saline (PBS; pH 7.2), before being embedded in paraffin and sectioned coronally with a microtome into $4-\mu \mathrm{m}$ sections. The tissue sections were then deparaffinized in xylol and hydrated in decreasing series of ethanol. Endogenous peroxidase activity was blocked by incubation in methanol containing $0.3 \% \mathrm{H}_{2} \mathrm{O}_{2}$ for $10 \mathrm{~min}$ at room temperature, followed by a rinse in $0.1 \mathrm{M} \mathrm{PBS}(\mathrm{pH} 7.4)$ for $5 \mathrm{~min}$. Before application of specific primary antibodies, nonspecific background staining was prevented by incubation with goat serum diluted 1:10 v/v in PBS for $50 \mathrm{~min}$. After incubation with either rabbit anti-enolase or anti-cav-1 (Santa Cruz Biotechnology, Inc., Santa Cruz, CA, USA) overnight at $4^{\circ} \mathrm{C}$, tissue sections were washed three times in PBS, and incubated with biotin-conjugated secondary antibody for $1 \mathrm{~h}$ at room temperature according to the manufacturer's instructions. After washing, tissue sections were incubated for $10 \mathrm{~min}$ in streptavidin-peroxidase and then washed three more times in PBS. Positive signals were visualized with diaminobenzidine, followed by counter-staining with methyl green as previously described (17-19). PBS was substituted for the primary antibody for the negative control.

Total integrated optical density (IOD), a parameter representing the expression levels of enolase and cav-1 in cardiac tissue, was determined using a cast-grid microscope (MetaMorph/DP10/Bx41, UIC/Olympus, US/JP) together with an image-analysis program (MetaMorph offline, version 4.65). Under magnification of $\mathrm{x} 400$, five images were captured for each immunostained section and the average IOD value was calculated as previously described $(18,19)$.

Statistical analysis. Statistical analysis was performed using the SPSS 11.0 software. The results are presented as the mean \pm SEM. The sources of variation were analyzed by the unpaired Student's t-test. $\mathrm{P}<0.05$ was considered to indicate a statistically significant difference.
Table I. IOD of enolase and caveolin-1 in the rat hearts.

\begin{tabular}{lcc}
\hline Groups & Enolase & Caveolin- 1 \\
\hline Control & $0.0053 \pm 0.00041$ & $0.0057 \pm 0.00053$ \\
Dexamethasone & $0.0121 \pm 0.00021$ & $0.0133 \pm 0.00062$
\end{tabular}

IOD, integrated optical density per field, which is proportional to the total amount of staining. Total enolase and caveolin-1 IOD expression in rats subjected to dexamethasone-induced toxicity was significantly higher compared to that in the control hearts $(\mathrm{P}<0.05$, respectively).

\section{Results}

Histological examination. Routine histological examination revealed little morphological changes in the rat hearts from each group (data not shown).

Immunohistochemical staining. Immunohistochemical staining was performed on formalin-fixed, paraffin-embedded sections from all parts of the heart including the conduction system.

Expression of enolase protein. Positive staining for the enolase antibody was detected mainly in the extracellular matrix, and the enolase antibody was manifested as fine brown granularity. Two observers examined the sections independently.

The photomicrographs in Fig. 1A and B show the positive expression of enolase in the control (Fig. 1A) and DEX-injured (Fig. 1B) rat hearts. Total IOD expression of enolase in the rat hearts from each group is shown in Table I. Total IOD expression of enolase in the rat heart following DEX-induced toxicity was significantly higher compared to the control (Table I, $\mathrm{P}<0.05)$.

Expression of caveolin-1 protein. Cav-1 staining was detected mainly in the extracellular matrix, and positive staining for cav-1 was manifested as fine brown granularity.

The distribution of cav-1 in the rat hearts of the control and DEX groups is shown in Fig. 2A and B, respectively. Total cav-1 IOD in the heart of animals subjected to DEX-induced toxicity was significantly higher compared to that in the control (Table. I, P<0.05).

\section{Discussion}

Although therapy with DEX is widely used for anti-inflammation treatment, its associated undesirable side effects markedly decrease the quality of life of patients.

Enolase is a multifunctional protein that participates in glycolysis and gluconeogenesis and acts as a plasminogen receptor on the cell surface of several organisms (20). It is a glycolytic enzyme that catalyzes the interconversion of phosphoenolpyruvate and 2-phosphoglycerate (21). It has been reported that enolase is a key glycolytic enzyme that catalyzes the dehydration of 2-phosphoglycerate to phosphoenolpyruvate (22). Furthermore, it has long been considered an enzyme of the glycolytic pathway ubiquitously occurring in the cytosol 
A

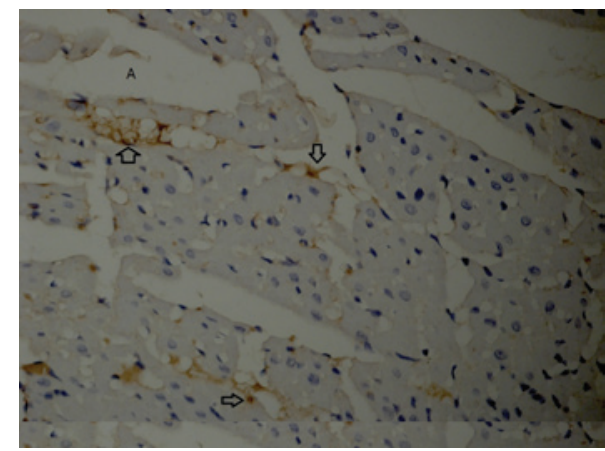

B

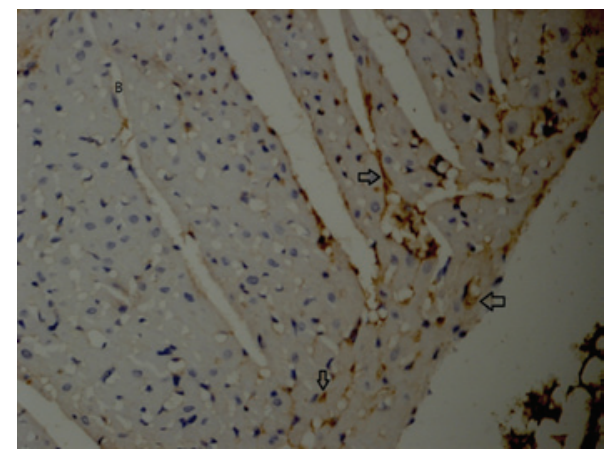

Figure 1. Effect of dexamethasone (DEX) exposure on enolase expression in the rat hearts. Photomicrographs display a representative distribution of enolase-positive expression in the (A) control and (B) DEX-exposed hearts. All photomicrographs were captured at a magnification of $\mathrm{x} 400$. Positive immunostaining appears as brown staining (arrow).

of prokaryotic and eukaryotic cells $(23,24)$. Recently, enolase has been shown to possess a variety of different regulatory functions, such as glycolysis, gluconeogenesis, hypoxia, ischemia and Alzheimer's disease (25). Aside from its classic role in carbohydrate metabolism, enolase was recently found to localize to membranes, where it binds host plasminogen and functions as a virulence factor for several pathogens (26). Also it has been demonstrated that enolase is an essential protein in fungal metabolism (27), and its lack of expression may cause delays in larval development (28).

Apart from its glycolytic function, enolase has also been shown to possess antigenic properties and to be present on the cell wall of certain invasive organisms, such as Candida albicans (29). In addition, it has been found that enolase levels are highly elevated in malaria parasite-infected red blood cells (30). Recently, enolase was revealed to be an important protein in many pathophysiological and disease processes (31). It is expressed on the surface of eukaryotic cells such as macrophages, neutrophils, endothelial, neuronal and tumor cells (32). Moreover, it is important in myogenesis, tumorgenesis and angiogenesis (32), as well as for pathogen invasiveness and the development of tumors (33). It has been shown that enolase mediates the activation of enzymes involved in the invasion of tissues by pathogens and tumor cells, as well as in the evasion of host immune responses (34), and that it is sensitive to fluoride (35). The increased amount of enolase in the heart noted in our study, suggests that the tissues were greatly damaged following DEX-induced toxicity. The present study indicates that DEX-induced toxicity causes an increase in enolase expression, which is related to impairment of the heart function.
A

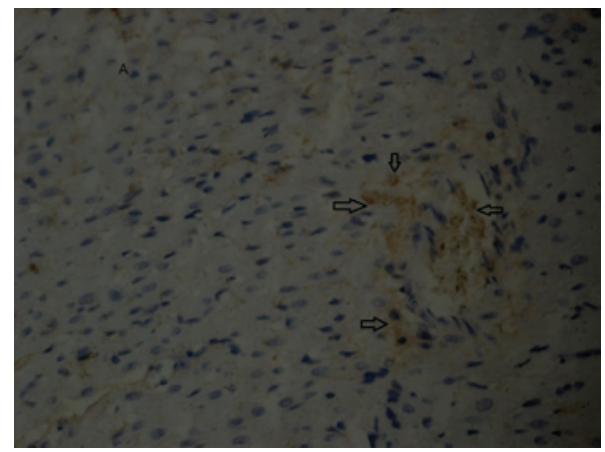

B

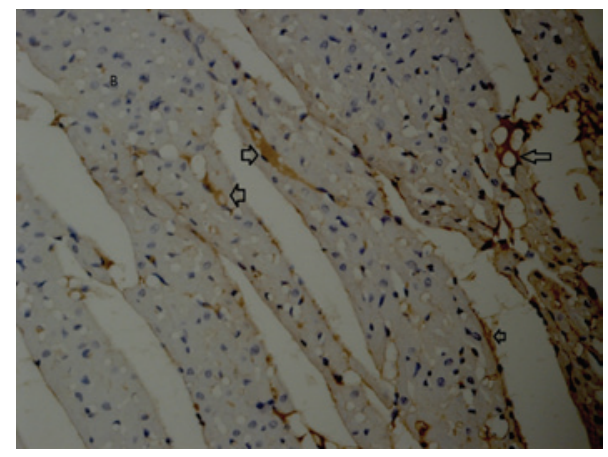

Figure 2. Effect of dexamethasone (DEX) exposure on caveolin-1 (cav-1) expression in the rat hearts. Photomicrographs reveal a representative distribution of positive cav-1 expression in the (A) control and (B) DEX-exposed hearts. All photomicrographs were captured at a magnification of x400. Positive immunostaining appears as brown staining (arrow).

Cav-1, a 21- to 24-kDa integral membrane protein, is a major structural coat protein of caveolae which are specialized plasma membrane invaginations involved in multiple cellular functions (36), including molecular transport, cell adhesion and signal transduction (37). In addition, cav-1 regulates critical cell functions including proliferation, apoptosis, cell differentiation and transcytosis via diverse signaling pathways (38). In addition, cav-1 is important for cell transformation, cell migration, metastasis and angiogenesis $(39,40)$. Furthermore, it is a membrane protein that forms the coat structure of plasma membrane caveolae and regulates caveola-dependent functions (41).

Cav-1 plays a crucial role in the regulation of various physiological and pathophysiological processes such as oncogenic transformation, tumorigenesis, tumor invasion and metastasis (42). Furthermore, it is a key protein involved in anoikis tumor metastasis (43), as well as a promising biomarker for identifying clinically significant cancer (37). It has been suggested that cav-1 may contribute to certain steps in carcinogenesis (44). It regulates multiple cancer-associated processes related to malignant tumor progression (45). Cav-1, which has been proposed as a candidate tumor suppressor, plays a regulatory role in several signaling pathways (46) and in the invasion of pathogenic microbes into the host cells (47). The increased cav-1 in the heart noted in our study suggests that the tissues were greatly damaged following DEX-induced toxicity. The present study indicates that DEX-induced toxicity causes an increase in cav-1 expression, which is related to impaired heart function.

In this research, our results demonstrate that cardiac protein expression of enolase and cav-1 is altered after DEX-induced toxicity in the rat. Following DEX-induced toxicity, protein 
expression of enolase and cav-1 was significantly elevated. The present study indicates that DEX-induced toxicity caused different alterations in heart proteins that are related to reduced cardiac function.

In conclusion, the present findings indicate that such alterations are reflected in abnormal cardiac function. Moreover, the proteins identified in this study may be useful for revealing the mechanisms underlying DEX-induced toxicity and also for providing various clues for further research.

\section{Acknowledgements}

This study was supported by a grant from the Natural Science Foundation of Guangdong Province, China (4203003) and the Guangdong Medical College (no. XQ0426).

\section{References}

1. Vanhaecke L, Antignac JP, Courtheyn D, Le Bizec B and De Brabander H: Elimination kinetics of dexamethasone in bovine urine, hair and feces following single administration of dexamethasone acetate and phosphate esters. Steroids 76: 111-117, 2011

2. Guerrero JA, Vicente V and Corral J: Dexamethasone induction of a heat stress response. Methods Enzymol 490: 121-135, 2011.

3. Ramessur S, Dinsmore J and Zoumprouli A: Hyperglycaemia and cerebral oedema in a patient with a meningioma receiving dexamethasone. Anaesthesia 66: 127-131, 2011.

4. Murphy GS, Szokol JW, Greenberg SB, et al: Preoperative dexamethasone enhances quality of recovery after laparoscopic cholecystectomy: effect on in-hospital and postdischarge recovery outcomes. Anesthesiology 114: 882-890, 2011.

5. Ghosn CR, Li Y, Orilla WC, et al: Treatment of experimental anterior and intermediate uveitis by a dexamethasone intravitreal implant. Invest Ophthalmol Vis Sci 52: 2917-2923, 2011.

6. Zakkar M, Luong le A, Chaudhury H, et al: Dexamethasone arterializes venous endothelial cells by inducing mitogenactivated protein kinase phosphatase-1: a novel antiinflammatory treatment for vein grafts? Circulation 123: 524-532, 2011.

7. Harel L, Prais D, Bar-On E, et al: Dexamethasone therapy for septic arthritis in children: results of a randomized double-blind placebo-controlled study. J Pediatr Orthop 31: 211-215, 2011.

8. Isoda A, Matsumoto M, Nakahashi H, Mawatari M, Manaka A and Sawamura M: Reduced risk of bacterial infection in multiple myeloma patients with VAD regimen without intermittent high-dose dexamethasone. Int J Hematol 93: 59-65, 2011.

9. Tsai YJ, Liang JG, Wu WB, Ding YF, Chiang RP and Wu SM: Intratympanic injection with dexamethasone for sudden sensorineural hearing loss. J Laryngol Otol 125: 133-137, 2011.

10. Liu Y and Van Der Leij FR: Long-term effects of neonatal treatment with dexamethasone, L-carnitine, and combinations thereof in rats. Pediatr Res 69: 148-153, 2011.

11. Mádi A, Majai G, Koy C, Vámosi G, Szántó A, Glocker MO and Fésüs L: Altered sialylation on the cell-surface proteins of dexamethasone-treated human macrophages contributes to augmented uptake of apoptotic neutrophils. Immunol Lett 135: 88-95, 2011.

12. Barnett CP, Chitayat D, Bradley TJ, Wang Y and Hinek A: Dexamethasone normalizes aberrant elastic fiber production and collagen 1 secretion by Loeys-Dietz syndrome fibroblasts: a possible treatment? Eur J Hum Genet 19: 624-633, 2011.

13. Heise N, Shumilina E, Nurbaeva MK, et al: Effect of dexamethasone on $\mathrm{Na}^{+} / \mathrm{Ca}^{2+}$ exchanger in dendritic cells. Am J Physiol Cell Physiol 300: C1306-C1313, 2011.

14. Mahmoud H, Mahmoud O, Layasadat $\mathrm{K}$ and Naeim A: Dexamethasone effects on Bax expression in the mouse testicular germ cells. Folia Histochem Cytobiol 47: 237-241, 2009.

15. Helin HO, Lundin ME, Laakso M, Lundin J, Helin HJ and Isola J: Virtual microscopy in prostate histopathology: simultaneous viewing of biopsies stained sequentially with hematoxylin and eosin, and alpha-methylacyl-coenzyme A racemase/p63 immunohistochemistry. J Urol 175: 495-499, 2006.

16. De Rossi A, Rocha LB and Rossi MA: Application of fluorescence microscopy on hematoxylin and eosin-stained sections of healthy and diseased teeth and supporting structures. J Oral Pathol Med 36: 377-381, 2007.
17. Smith PS, Parkinson IH and Leong AS: Principles of ploidy analysis by static cytometry. Clin Mol Pathol 49: M104-M111, 1996.

18. Dong J, Yin H, Liu W, Wang P, Jiang Y and Chen J: Congenital iodine deficiency and hypothyroidism impair LTP and decrease $\mathrm{C}$-fos and $\mathrm{C}$-jun expression in rat hippocampus. Neurotoxicology 26: 417-426, 2005.

19. van Kuijk AW, Gerlag DM, Vos K, et al: A prospective, randomised, placebo-controlled study to identify biomarkers associated with active treatment in psoriatic arthritis: effects of adalimumab treatment on synovial tissue. Ann Rheum Dis 68: 1303-1309, 2009.

20. Sánchez-Miguel DS, Romero-Jiménez J, Reyes-López CA, Cabrera-Avila AL, Carrillo-Ibarra N and Benítez-Cardoza CG: Chemical unfolding of enolase from Saccharomyces cerevisiae exhibits a three-state model. Protein J 29: 1-10, 2010.

21. Yamamoto $\mathrm{H}$ and Kunishima N: Purification, crystallization and preliminary crystallographic study of the putative enolase MJ0232 from the hyperthermophilic archaeon Methanococcus jannaschii. Acta Crystallogr Sect F Struct Biol Cryst Commun 64: 1087-1090, 2008.

22. Zhou W, Capello M, Fredolini C, Piemonti L, Liotta LA, Novelli F and Petricoin EF: Mass spectrometry analysis of the post-translational modifications of alpha-enolase from pancreatic ductal adenocarcinoma cells. J Proteome Res 9: 2929-2936, 2010.

23. Seweryn E, Pietkiewicz J, Szamborska A and Gamian A: Enolase on the surface of prockaryotic and eukaryotic cells is a receptor for human plasminogen. Postepy Hig Med Dosw (Online) 61: 672-682, 2007 (In Polish).

24. Vanegas G, Quiñones W, Carrasco-López C, Concepción JL, Albericio F and Avilán L: Enolase as a plasminogen binding protein in Leishmania mexicana. Parasitol Res 101: 1511-1516, 2007.

25. Butterfield DA and Lange ML: Multifunctional roles of enolase in Alzheimer's disease brain: beyond altered glucose metabolism. J Neurochem 111: 915-933, 2009.

26. Swenerton RK, Zhang S, Sajid M, Medzihradszky KF, Craik CS, Kelly BL and McKerrow JH: The oligopeptidase B of Leishmania regulates parasite enolase and immune evasion. J Biol Chem 286: 429-440, 2011.

27. Gandhi NS, Young K, Warmington JR and Mancera RL: Characterization of sequence and structural features of the Candida krusei enolase. In Silico Biol 8: 449-460, 2008.

28. Chen N, Xu MJ, Nisbet AJ, et al: Ascaris suum: RNAi mediated silencing of enolase gene expression in infective larvae. Exp Parasitol 127: 142-146, 2011.

29. Pal-Bhowmick I, Mehta M, Coppens I, Sharma S and Jarori GK: Protective properties and surface localization of Plasmodium falciparum enolase. Infect Immun 75: 5500-5508, 2007.

30. Pal-Bhowmick I, Vora HK and Jarori GK: Sub-cellular localization and post-translational modifications of the Plasmodium yoelii enolase suggest moonlighting functions. Malar J 6: 45, 2007.

31. Wang J, Zhou YF, Li LF and Su XD: Crystallization and preliminary X-ray analysis of human liver alpha-enolase. Acta Crystallogr Sect F Struct Biol Cryst Commun 65: 288-290, 2009.

32. Seweryn E, Bednarz-Misa IS, Danielewicz R, et al: Distribution of beta-enolase in normal and tumor rat cells. Folia Histochem Cytobiol 46: 519-524, 2008.

33. Seweryn E, Pietkiewicz J, Bednarz-Misa IS, et al: Localization of enolase in the subfractions of a breast cancer cell line. Z Naturforsch C 64: 754-758, 2009.

34. Falabella P, Riviello L, De Stradis ML, et al: Aphidius ervi teratocytes release an extracellular enolase. Insect Biochem Mol Biol 39: 801-813, 2009.

35. van Loveren C, Hoogenkamp MA, Deng DM and ten Cate JM: Effects of different kinds of fluorides on enolase and ATPase activity of a fluoride-sensitive and fluoride-resistant Streptococcus mutans strain. Caries Res 42: 429-434, 2008.

36. Tsai TH, Chen SF, Huang TY, et al: Impaired Cd14 and Cd36 expression, bacterial clearance, and Toll-like receptor 4-Myd88 signaling in caveolin-1-deleted macrophages and mice. Shock 35: 92-99, 2011.

37. Corn PG and Thompson TC: Identification of a novel prostate cancer biomarker, caveolin-1: implications and potential clinical benefit. Cancer Manag Res 2: 111-122, 2010.

38. Jin Y, Lee SJ, Minshall RD and Choi AM: Caveolin-1: a critical regulator of lung injury. Am J Physiol Lung Cell Mol Physiol 300: L151-L160, 2011. 
39. Li M, Chen H, Diao L, Zhang Y, Xia C and Yang F: Caveolin-1 and VEGF-C promote lymph node metastasis in the absence of intratumoral lymphangiogenesis in non-small cell lung cancer. Tumori 96: 734-743, 2010.

40. Wang X, Feng S, Zhang H, et al: RNA inference-mediated caveolin-1 down-regulation decrease estrogen receptor alpha (ERalpha) signaling in human mammary epithelial cells. Mol Biol Rep 38: 761-768, 2011.

41. Ravid D, Leser GP and Lamb RA: A role for caveolin 1 in assembly and budding of the paramyxovirus parainfluenza virus 5. J Virol 84: 9749-9759, 2010.

42. Zhu H, Yue J, Pan Z, et al: Involvement of Caveolin-1 in repair of DNA damage through both homologous recombination and non-homologous end joining. PLoS One 5: e12055, 2010.

43. Rungtabnapa P, Nimmannit U, Halim H, Rojanasakul Y and Chanvorachote P: Hydrogen peroxide inhibits non-small cell lung cancer cell anoikis through the inhibition of caveolin-1 degradation. Am J Physiol Cell Physiol 300: C235-C245, 2011.
44. Syeed N, Husain SA, Abdullah S, et al: Caveolin-1 promotes mammary tumorigenesis: mutational profile of the Kashmiri population. Asian Pac J Cancer Prev 11: 689-696, 2010.

45. Sáinz-Jaspeado M, Lagares-Tena L, Lasheras J, et al: Caveolin-1 modulates the ability of Ewing's sarcoma to metastasize. Mol Cancer Res 8: 1489-1500, 2010.

46. Bau DT, Tsai MH, Tsou YA, et al: The association of Caveolin-1 genotypes with oral cancer susceptibility in Taiwan. Ann Surg Oncol 18: 1431-1438, 2011.

47. Liu XD, Chen HB, Tong Q, et al: Molecular characterization of Caveolin-1 in pigs infected with Haemophilus parasuis. J Immunol 186: 3031-3046, 2011. 